\section{ORIGINAL RESEARCH}

\author{
S. Ropele \\ C. Enzinger \\ M. Söllinger \\ C. Langkammer \\ M. Wallner-Blazek \\ R. Schmidt \\ F. Fazekas
}

\title{
The Impact of Sex and Vascular Risk Factors on Brain Tissue Changes with Aging: Magnetization Transfer Imaging Results of the Austrian Stroke Prevention Study
}

BACKGROUND AND PURPOSE: Quantitative MR imaging techniques allow detection of subtle tissue changes that occur with brain aging beyond the accumulation of $\mathrm{WMH}$ and brain atrophy. To what extent sex and cerebrovascular risk factors impact these changes is largely unknown. We attempted to study these risk factors in the context of the community-based ASPS.

MATERIAL AND METHODS: We performed MTI in 328 neurologically asymptomatic ASPS participants (age range, 52-87 years). FLAIR was used to delineate WMH and to define NABT. The MTR was measured globally in NABT by using a histogram analysis technique and focally in WMH. Associations of MTR metrics with sex and a large battery of different cerebrovascular risk factors (age, arterial hypertension, diabetes mellitus, smoking, body mass index, cholesterol and triglyceride levels, glycated hemoglobin, and the presence of cardiac disease) were assessed with univariate and multiple regression analysis.

RESULTS: Age was seen to affect all MTR histogram metrics of NABT, and a faster decrease of the MTR peak height occurred in men. Independent associations with MTR metrics were found for arterial hypertension and diabetes mellitus. Besides lesion grade, arterial hypertension was also significantly associated with a lower MTR in WMH.

CONCLUSIONS: Microstructural tissue changes of NABT increase with aging and may be more extensive in men. Diabetes mellitus and hypertension appear to add to tissue destruction. The exact mechanisms involved await further clarification.

\begin{abstract}
ABBREVIATIONS: ASPS = Austrian Stroke Prevention Study; BMI = body mass index; DTI = diffusion tensor imaging; FLAIR = fluid-attenuated inversion recovery; FWHM = full width at half maximum; $\mathrm{Hb}_{1 \mathrm{c}}^{\mathrm{A}}=$ glycol hemoglobin; $\mathrm{HDL}=$ high-density lipoprotein; max = maximum; $\mathrm{MT}=$ magnetization transfer; $\mathrm{MTI}=\mathrm{MT}$ imaging; $\mathrm{MTR}=\mathrm{MT}$ ratio; $\mathrm{NABT}=$ normal-appearing brain tissue; $\mathrm{WMH}=$ white matter hyperintensity
\end{abstract}

$\mathbf{M}$ $\mathrm{R}$ imaging has shown that the aging brain-apart from overt disease-can undergo various changes that may have a significant impact on cognitive function, gait, balance, and mood. Among those readily appreciated on conventional MR imaging are the accumulation of WMH, silent lacunes and infarcts, and cerebral atrophy. ${ }^{1-4}$ The contribution of cerebrovascular risk factors to these alterations has been assessed in several studies. ${ }^{5-8}$ From MR imaging techniques such as DTI or MTI probing tissue composition and microstructure, there is evidence for additional more subtle changes that may occur with aging of the brain. ${ }^{9-12}$ These can also bear clinical relevance because of the large volume of potentially affected but NABT and as possible precursors of gross morphologic damage. ${ }^{13,14}$ So far the pathophysiologic background of these microstructural alterations, such as their association with cere-

Received November 12, 2009; accepted after revision December 29.

From the Department of Neurology, Medical University of Graz, Graz, Austria.

This work was supported by the Austrian Science Foundation (P20103).

Please address correspondence to Stefan Ropele, PhD, Department of Neurology, Medical University of Graz, Auenbruggerplatz 22, A-8036 Graz, Austria; e-mail: stefan.ropele@ medunigraz.at

Indicates open access to non-subscribers at www.ajnr.org

DOI 10.3174/ajnr.A2042

brovascular risk factors and their relation to sex, has not been studied in detail, to our knowledge.

MTI has been established as an important tool for detecting microstructural tissue changes in inflammatory and neurodegenerative diseases of the brain. ${ }^{15}$ MTI is based on the exchange of magnetization between tissue water and protons that are bound to macromolecules such as the myelin lipids and proteins. The rate of exchange gives an estimate of the magnitude of these compartments, such as the pool of bound protons, which cannot be measured directly by conventional MR imaging due to their extremely short $\mathrm{T} 2$ relaxation time. ${ }^{16}$ The efficacy of the MT is usually quantified by the MTR. In brain tissue, the MTR correlates with macromolecular attenuation and, therefore, is believed to largely reflect myelin content. Supportive evidence for this concept comes from basic models of $\mathrm{MT}^{17}$ and more recently from correlative histopathologic studies. ${ }^{18}$ Water content and other components of brain tissue are likely to contribute to MTR changes as well.

It is well established that the MTR increases as a consequence of myelination and brain maturation during the first 2 years of life. ${ }^{19,20}$ Thereafter it is thought to remain constant until the last decades of life, when a strong negative correlation of the MTR with age has been reported. ${ }^{21-23}$ Some sex-related differences confined to specific brain regions have also been suggested. ${ }^{24}$ Whether and to what extent additional factors 
may influence the MTR of brain tissue with aging is widely unknown. In an extension of an earlier study that focused on $\mathrm{WMH},{ }^{10}$ we examined the association of sex and vascular risk factors with cerebral MTR metrics in a large cohort of healthy elderly individuals.

\section{Materials and Methods}

\section{Subjects}

The study cohort was drawn from the ASPS, which is a prospective single-center community-based follow-up study with the goal of examining the frequency of vascular risk factors and their effects on cerebral morphology and function in the healthy elderly. ${ }^{25}$ An invitation to the study followed random selection from the community register. On the basis of a structured clinical interview and a physical and neurologic examination, participants had to be free of overt neurologic or psychiatric findings and had to have no history of a neuropsychiatric disease, including previous cerebrovascular attacks and dementia. The study protocol was approved and accepted by the ethics committee of the Medical University of Graz, Austria, and informed consent was obtained from all study participants.

The following demographic variables and risk factors were considered in the current study: age, sex, systolic blood pressure, diastolic blood pressure, a diagnosis of arterial hypertension and diabetes mellitus, smoking, the body mass index, cholesterol and triglyceride levels, glycated hemoglobin, and the presence of cardiac disease comprising sources such as cardiac embolism, coronary heart disease, and left ventricular hypertrophy. Arterial hypertension was considered present if there was a history of arterial hypertension with repeated blood pressure readings higher than $160 / 95 \mathrm{~mm} \mathrm{Hg}$, if an individual was treated for arterial hypertension, or if the 3 readings of the systolic and diastolic blood pressure exceeded this limit. Diabetes mellitus was coded as present if a subject was treated for diabetes at the time of examination or if the fasting blood glucose level at 1 examination exceeded $140 \mathrm{mg} / \mathrm{dL}$. With regard to smoking, subjects were grouped into nonsmokers, former smokers, and current (long-term) smokers.

\section{MR Imaging}

MR imaging was performed on a 1.5T ACS-Intera system (Philips Healthcare, Best, the Netherlands) with a protocol that included a T2-weighted fast spin-echo sequence and a FLAIR sequence. Section thickness was $5 \mathrm{~mm}$ with a $0.5-\mathrm{mm}$ intersection gap. At a later stage of the ASPS study, MTI was added to the scanning protocol and was performed in 372 subjects. MTI was performed after conventional MR imaging with a spoiled $3 \mathrm{D}$ gradient-echo sequence $(\mathrm{TE}=4 \mathrm{~ms}$, $\mathrm{TR}=26 \mathrm{~ms}, \mathrm{FA}=20^{\circ}$, section thickness $=3 \mathrm{~mm}, \mathrm{FOV}=250 \mathrm{~mm}$, matrix $=256 \times 256)$ with and without a binomial saturation pulse $\left(1-2-1, B_{1 \max }=21 \mu \mathrm{T}\right)$. The axial sections of the MT and FLAIR sequences covered the whole brain and were positioned to run parallel to a line defined by the most inferoanterior and the most inferoposterior parts of the corpus callosum.

\section{Image Analysis}

MTR metrics were assessed separately for the NABT by means of histogram analysis and for WMH by calculating the mean lesional MTR. The definition of NABT was based on a normal signal intensity on FLAIR images (ie, it comprised all brain tissue outside WMHs). For this purpose, WMHs were first specified and graded by a single rater (C.E.), according to our scheme, into absent, punctate, early confluent, and confluent. ${ }^{26}$ They were then outlined semiautomati- cally with the program DispImage (provided by Dave Plummer, University College of London, London, UK) to generate corresponding WMH masks and to calculate a WMH volume for every subject.

MTR maps were calculated according to the formula MTR = $\left(\mathrm{M}_{\mathrm{ss}}-\mathrm{M}_{0}\right) / \mathrm{M}_{0}$, where $\mathrm{M}_{\mathrm{ss}}$ and $\mathrm{M}_{0}$ are the signal intensities obtained with and without MT saturation, respectively. The MTR maps then were registered with the FLAIR scans by using an automated affine registration tool (FLIRT as part of FSL, http://www.fmrib.ox.ac.uk/ fsl/flirt/index.html).

A mean MTR was calculated for each WMH by masking the registered MTR maps with the WMH masks. To reduce partial volume effects, which might have taken place due to image registration and subsequent interpolation, we eroded all masks by 1 pixel. The MTRs of all WMHs were averaged to obtain a mean lesional MTR for each subject.

To analyze the MTR metrics of NABT, we removed all WMH areas from the MTR maps after dilating the original WMH masks by 1 pixel and removed nonbrain tissue with a brain extraction tool (BET as part of FSL, http://www.fmrib.ox.ac.uk/fsl/bet2/index.html). All remaining voxels in the MTR maps were then considered for the MTR histogram analysis. For each histogram, we calculated the following: 1) the peak position (ie, the MTR value with the highest frequency), 2) the relative peak height (ie, the relative voxel count at the peak position), and 3) the FWHM. To correct for differences in individual brain volumes, we normalized the histograms by the total number of voxels contributing to the histogram.

For the present analysis, we excluded 44 subjects from the image analysis for the following reasons: severe motion artifacts on FLAIR or MTI scans $(n=26)$, silent chronic brain infarcts $(n=8)$, single large lesions $(>20 \mathrm{~mm}$ ) or diffuse white matter changes of uncertain etiology $(n=8)$, or hydrocephalus $(n=2)$.

\section{Statistical Analysis}

The primary goal of this study was to probe the impact of sex and different cerebrovascular risk factors on microstructural brain changes assessed with MTI. We used the Statistical Package for Windows (StatSoft, Tulsa, Oklahoma) for data analysis. First, univariate analysis was used for descriptive statistics of the male and female participants and to identify potential risk factors that may have an effect on mean lesional (WMH) MTR and on the MTR metrics of the NABT (ie, the histogram peak height, position, and FWHM). To investigate the effect of sex, hypertension, smoking, cardiac disease, and diabetes, we used a Student $t$ test for independent samples. A 1-way analysis of variance was used to assess the effect of WMH severity on the MTR indices. For this purpose, we grouped individuals according to the following WMH characteristics: $0-2$ punctate WMHs, $>2$ punctate WMHs, early confluent WMHs, and confluent WMHs. For all other variables, a linear regression analysis was used. Finally, a multiple regression analysis was used to identify those factors that independently impacted MTR indices. The regression analysis was performed for the total study cohort by using sex as an independent factor. A $P$ value $<.05$ was considered significant.

\section{Results}

\section{Subjects}

The final study cohort consisted of 328 individuals without significant MR imaging pathologies with adequate FLAIR and MTI scans. There were 215 women and 113 men with a mean age of 70.5 years, ranging from 52.7 to 87.2 years. Sex sub- 


\begin{tabular}{lccc}
\hline \multicolumn{4}{l}{ Table 1: Characteristics of investigated cohort } \\
\hline & Men & Women & All \\
& $(n=113)$ & $(n=215)$ & $(n=328)$ \\
\hline Age (years) & $70.1(6.0)$ & $70.7(6.2)$ & $70.5(6.1)$ \\
WMH score 1 (\%) & 54.8 & 63.7 & 60.6 \\
WMH score 2 (\%) & 19.4 & 14.8 & 16.4 \\
WMH score 3 (\%) & 9.7 & 10.2 & 10.0 \\
WMH volume (cm $\left.{ }^{3}\right)$ & $6.7(12.6)$ & $6.0(10.1)$ & $6.2(11.0)$ \\
Hypertension (\%) & 60.1 & 51.6 & 54.5 \\
Hb ${ }_{1 \mathrm{c}}$ (mg/dL) & $5.60(0.68)$ & $5.63(0.61)$ & $5.62(0.64)$ \\
Diabetes (\%) & 12.3 & 8.4 & 9.7 \\
Current and former smokers (\%) & 60.1 & 21.8 & $44.8^{\mathrm{a}}$ \\
Cardiac disease (\%) & 33.6 & 31.1 & 32.0 \\
BMI (kg/m²) & $26.7(2.9)$ & $26.6(4.8)$ & $26.6(4.3)$ \\
HDL (mg/dL) & $51.1(11.5)$ & $63.3(16.7)$ & $58.9(16.1)^{\mathrm{a}}$ \\
Total cholesterol (mg/dL) & $220.2(39.4)$ & $232.1(42.9)$ & $227.7(41.9)^{\mathrm{a}}$ \\
Triglycerides (mg/dL) & $130.5(57.7)$ & $110.0(47.5)$ & $117.6(52.3)^{\mathrm{a}}$ \\
\hline a Significant differences between men and women $(P<.05)$. &
\end{tabular}

Table 2: Effect of risk factors on global and lesional MTR metrics ${ }^{a}$

\begin{tabular}{|c|c|c|c|c|}
\hline & \multicolumn{3}{|c|}{ NABT } & \multirow{2}{*}{$\begin{array}{c}\frac{\text { WMH }}{\text { Mean }} \\
\text { MTR }\end{array}$} \\
\hline & $\begin{array}{c}\text { Peak } \\
\text { Position }\end{array}$ & $\begin{array}{l}\text { Peak } \\
\text { Height }\end{array}$ & FWHM & \\
\hline$\overline{\text { Age }}$ & $-0.32^{b}$ & $-0.21^{\mathrm{b}}$ & $0.15^{b}$ & -0.18 \\
\hline WMH volume & $-0.19^{b}$ & $-0.19^{b}$ & 0.10 & $-0.24^{b}$ \\
\hline Systolic pressure & $-0.14^{b}$ & -0.08 & 0.08 & -0.12 \\
\hline Diastolic pressure & 0.00 & -0.01 & -0.01 & 0.03 \\
\hline $\mathrm{Hb}_{1 \mathrm{c}}^{\mathrm{A}}$ & -0.13 & $-0.18^{\mathrm{b}}$ & 0.06 & -0.07 \\
\hline $\mathrm{BMI}$ & -0.06 & -0.10 & 0.07 & 0.02 \\
\hline HDL & 0.10 & 0.11 & -0.16 & 0.11 \\
\hline Total cholesterol & -0.04 & -0.06 & 0.02 & -0.03 \\
\hline Triglycerides & -0.13 & -0.15 & 0.11 & -0.07 \\
\hline
\end{tabular}

${ }^{a}$ The correlation factors of the univariate correlation analysis are shown.

${ }^{\mathrm{b}}$ Significant correlation $(P<.05)$.

groups were comparable for age and WMH severity, but women smoked significantly less and had higher cholesterol levels and lower levels of triglycerides (Table 1).

\section{Predictors of the MTR of NABT}

Sex Effects. Univariate analyses of the entire cohort revealed age as the strongest significant predictor for MTR histogram metrics in NABT (Table 2). In a separate regression analysis for men and women, the peak position of the MTR histogram was shifted toward lower MTR values with increasing age in both sexes (Fig 1). In relation to the relative peak height, men showed a more rapid decline with age than women. The regression function could be modeled best by a second order polynomial and revealed that this sex difference started between 60 and 65 years of age (Fig 2).

Effects of Cerebrovascular Risk Factors. Univariate analysis of vascular risk factors on MTR metrics showed significant associations with MTR peak height and position in NABT for WHM volume; systolic blood pressure; the level of glycated hemoglobin; and the presence of hypertension, diabetes mellitus, and cardiac disease (Tables 2 and 3). WMH severity affected the peak height only when it was seen to decrease with higher WMH grades. With significant predictors in a linear regression model, only age, sex, diabetes, and hypertension remained as independent factors for MTR changes in NABT (Table 4). Using the FWHM did not reveal any association beyond those seen with the peak height and the peak position.

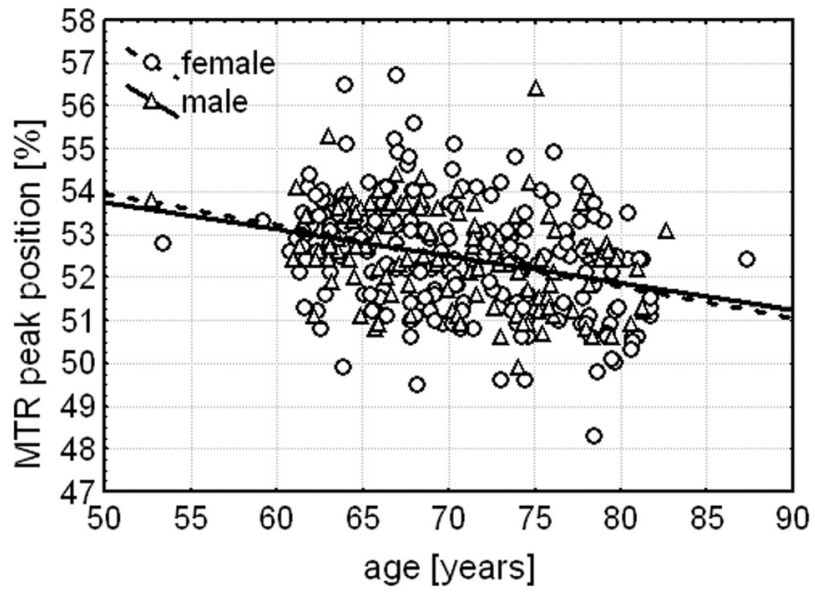

Fig 1. MTR peak position in NABT as a function of age. Men and women show a similar slope of decline over the investigated age range.

\section{Predictors of the MTR of WMHs}

The mean MTR of WMH was significantly lower than the histogram peak position (ie, the MTR of normal-appearing white matter). The only factors that independently affected lesional MTR were WMH grade and arterial hypertension. Accordingly, subjects with hypertension had a significantly lower lesional (WMH) MTR than normotensive subjects (Table 3 and Fig 3). Systolic or diastolic blood pressure did not correlate with the lesional MTR.

\section{Discussion}

This is the first study that assessed brain tissue changes in a large cohort of healthy elderly individuals by using MTI. Our results confirm a significant impact of aging per se on the composition of NABT. As a novel finding, we also identified modulatory effects of sex, hypertension, and diabetes mellitus on MTR metrics. In contrast, MTR changes in WMHs were related only to hypertension and WMH severity and not to the individual's age. In this context, the MTR metrics of NABT will be governed almost exclusively by contributions from white matter. The reason for this is a much higher MTR of white than gray matter. As a consequence, the prevailing decrease of gray matter tissue with aging and the ensuing shift in gray/white matter proportions may only partly have influenced these results. Even in the case of gray matter changes or atrophy, the peak position of NABT remains unchanged because it reflects essentially the mean MTR of white matter, provided the histogram is bimodal. ${ }^{27,28}$ Thus, it is commonly believed that a shift of the peak position toward lower MTR values implies diffuse changes in white matter but no changes in the gray/white matter ratio. In contrast, the relative peak height of the histogram reflects the relative number of voxels in normal white matter in relation to the total number of voxels contributing to the histogram. A decrease of the peak height of the MTR histogram thus indicates just a reduction of the amount of normal white matter within NABT. This can be caused by focal tissue destruction and any change in the proportion of tissue compartments (ie, also a change in the proportions of white matter, gray matter, and CSF compartments).

The observed changes in MTR histogram parameters of the 


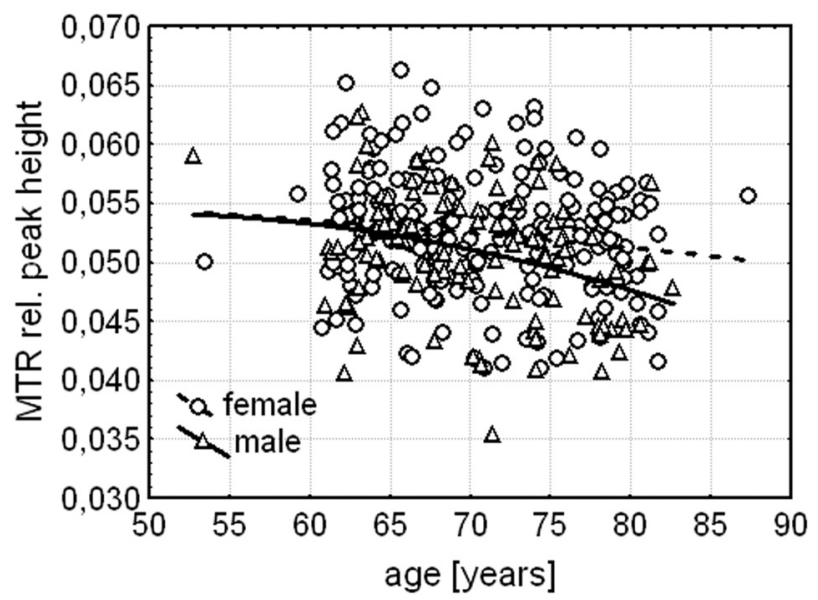

Fig 2. Relative peak height from the MTR histogram analysis in NABT. From between 60 and 65 years onwards, men show a steeper age-dependent decline than women.

Table 3: Effect of sex and dichotomized risk factors on global and lesional MTR metrics ${ }^{a}$

\begin{tabular}{|c|c|c|c|c|}
\hline & \multicolumn{3}{|c|}{ NABT } & \multirow{2}{*}{$\begin{array}{c}\text { WMH } \\
\text { Mean } \\
\text { MTR }\end{array}$} \\
\hline & $\begin{array}{c}\text { Peak } \\
\text { Position }\end{array}$ & $\begin{array}{l}\text { Peak } \\
\text { Height }\end{array}$ & FWHM & \\
\hline$\overline{S e x}$ & 0.268 & $-2.638^{b}$ & $3.908^{b}$ & 0.191 \\
\hline Hypertension & 2.792 & 0.979 & 0.440 & $2.018^{\mathrm{b}}$ \\
\hline Diabetes & 1.468 & $2.126^{b}$ & -0.603 & 0.451 \\
\hline Smoking & -1.499 & -1.107 & 1.417 & -1.020 \\
\hline Cardiac disease & -1.766 & $-2.628^{b}$ & 0.321 & -0.485 \\
\hline
\end{tabular}

a The $t$ values of an unpaired $t$ test are shown.

${ }^{\mathrm{b}}$ Significant factor $(P<.05)$.

\begin{tabular}{|c|c|c|c|c|c|c|c|c|c|}
\hline \multirow{2}{*}{$\begin{array}{l}\text { MTR in } \\
\text { NABT }\end{array}$} & \multicolumn{2}{|c|}{ Age } & \multicolumn{2}{|c|}{ Sex } & \multicolumn{2}{|c|}{ Hypertension } & \multicolumn{2}{|c|}{ Diabetes } & \multirow[b]{2}{*}{$r$} \\
\hline & $\beta$ & $P$ & $\beta$ & $P$ & $\beta$ & $P$ & $\beta$ & $P$ & \\
\hline Peak position & -0.32 & $<.01$ & & & -0.12 & $<.01$ & & & 0.35 \\
\hline Peak height & -0.20 & $<.01$ & -0.14 & $<.01$ & & & -.11 & .04 & 0.27 \\
\hline FWHM & 0.10 & .04 & 0.22 & $<.01$ & & & & & \\
\hline
\end{tabular}

${ }^{a}$ Effect size and $P$ value are shown.

NABT with increasing age, which were seen in regard to both peak position and peak height, were not unexpected and have already been reported in previous studies. ${ }^{10,21-23}$ Yet, some studies failed to demonstrate a significant age effect. ${ }^{9,29}$ This difference may be explained by the fact that some of these investigations included cohorts with a broader age range and did not correct for the plateauing during midlife. Another possible explanation is differences in the applied MT protocol.

The size and selection criteria of the investigated cohort also allowed exploration of the impact of sex and vascular risk factors on MTR metrics with normal aging. We thus observed that the MTR peak height declined with age more steeply in men than in women while the histogram peak position was not affected by sex. This sex difference cannot be explained by different risk profiles in men and women because those factors that were significantly different between sexes did not show a correlation with MTR metrics. Furthermore, a significant effect of sex was retained in the multivariate regression model. The sex difference was seen only in the peak height and not in the peak position. This finding suggests a more rapid loss of

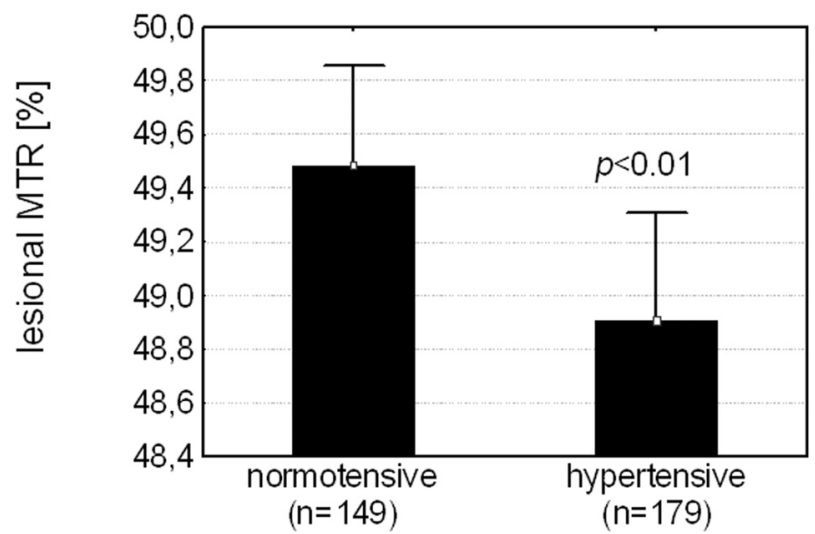

Fig 3. Hypertensive subjects (mean age, 71.2 years) had a significantly lower lesional MTR than normotensive subjects (mean age, 69.6 years). The whiskers represent the standard error

normal white matter with aging in men. While it could be speculated that more pronounced atrophy of gray matter structures may have contributed to this finding, this is unlikely (ie, greater loss of gray matter-which has been reported by some to occur in men ${ }^{30-32}$ - should have left the peak height unaffected rather than caused a decrease because of the increasing relative contribution of the white matter compartment in such a scenario). A recent study using multifrequency MR elastography, which reported a more preserved viscoelasticity of the brain with aging in women than men, is in line with our findings. ${ }^{33}$

Multiple regression analysis revealed diabetes mellitus and hypertension as independent predictors for MTR reductions in the NABT. Both have been associated with changes in microperfusion and damage of the blood-brain barrier, and both are risk factors for $\mathrm{WMH}$ and atrophy. ${ }^{34-38}$ Our finding that the presence of diabetes reduces histogram peak height while preserving the mean MTR value in the white matter (ie, the MTR peak position), most likely reflects brain tissue loss rather than demyelination or global changes of the microstructure. In contrast, hypertension affected the peak position only, which means that changes related to hypertension were more likely global and diffuse in nature. Regarding focal damage (ie, WMH), we confirmed lesion grade and hypertension as predictors of more severe tissue damage. ${ }^{10}$ 
The strengths of this study are the large and homogeneous study cohort and the use of a very sensitive MT protocol, which was achieved by a $3 \mathrm{D}$ sequence with a high repetition rate of the MT saturation pulses. The latter is also substantiated by the high MTR values observed, though so-called direct saturation effects produced by the binomial pulses might also have contributed to this. ${ }^{39}$ A limitation certainly comes from the fact that we were able to explain only a relatively small portion of the variability of the MTR metrics despite the large number of variables assessed. For specific information on gray and white matter structures, it would have also been desirable to assess different tissue compartments separately. However, because the scans were acquired at a time when tissue segmentation was not yet a consideration, we abstained from such efforts.

\section{Conclusions}

We here confirm MTI as a sensitive tool for the depiction of microstructural changes with aging in otherwise NABT. From our results, there is also evidence for diffusely damaging effects of hypertension while male sex and diabetes mellitus may contribute to a more rapid loss of normal white matter. The biochemical and histopathologic correlates of observed MTR changes and the pathophysiologic mechanisms involved await further clarification.

\section{References}

1. de Leeuw FE, de Groot JC, Achten E, et al. Prevalence of cerebral white matter lesions in elderly people: a population based magnetic resonance imaging study-The Rotterdam Scan Study. J Neurol Neurosurg Psychiatry 2001; 70:9-14

2. Van Dijk EJ, Prins ND, Vermeer SE, et al. Frequency of white matter lesions and silent lacunar infarcts. J Neural Transm Suppl 2002;62:25-39

3. Blatter D, Bigler E, Gale S, et al. Quantitative volumetric analysis of brain MR: normative database spanning $\mathbf{5}$ decades of life. AJNR Am J Neuroradiol 1995;16:241-51

4. Good CD, Johnsrude IS, Ashburner J, et al. A voxel-based morphometric study of ageing in 465 normal adult human brains. Neuroimage 2001;14(1 pt 1): 21-36

5. Den Heijer T, Launer LJ, Prins ND, et al. Association between blood pressure, white matter lesions, and atrophy of the medial temporal lobe. Neurology 2005;64:263-67

6. Schmidt R, Fazekas F, Kapeller P, et al. MRI white matter hyperintensities: three-year follow-up of the Austrian Stroke Prevention Study. Neurology 1999;53:132-39

7. Enzinger C, Fazekas F, Matthews PM, et al. Risk factors for progression of brain atrophy in aging: six-year follow-up of normal subjects. Neurology 2005;64: $1704-11$

8. Sachdev P. Homocysteine, cerebrovascular disease and brain atrophy. J Neurol Sci 2004;15;226:25-29

9. Rovaris M, Iannucci G, Cercignani M, et al. Age-related changes in conventional, magnetization transfer, and diffusion-tensor MR imaging findings: study with whole-brain tissue histogram analysis. Radiology 2003;227:731-38

10. Fazekas F, Ropele S, Enzinger C, et al. MTI of white matter hyperintensities. Brain 2005;128:2926-32

11. Vernooij MW, de Groot M, van der Lugt A, et al. White matter atrophy and lesion formation explain the loss of structural integrity of white matter in aging. Neuroimage 2008;43:470-77

12. Bennett IJ, Madden DJ, Vaidya CJ, et al. Age-related differences in multiple measures of white matter integrity: a diffusion tensor imaging study of healthy aging. Hum Brain Mapp 2010;31:378-90

13. Charlton RA, Barrick TR, McIntyre DJ, et al. White matter damage on diffusion tensor imaging correlates with age-related cognitive decline. Neurology 2006;66:217-22

14. Taylor WD, Bae JN, MacFall JR, et al. Widespread effects of hyperintense lesions on cerebral white matter structure. AJR Am J Roentgenol 2007;188: 1695-704

15. Tofts P, Steens S, van Buchem M. MT: magnetization transfer. In: Tofts P, ed. Quantitative MRI of the Brain. Chichester, UK: John Wiley \& Sons; 2003:257-98

16. Wolff SD, Balaban RS. Magnetization transfer contrast (MTC) and tissue water proton relaxation in vivo. Magn Reson Med 1989;10:135-44

17. Morrison C, Henkelman RM. A model for magnetization transfer in tissues. Magn Reson Med 1995;33:475-82

18. Schmierer K, Scaravilli F, Altmann D, et al. Magnetization transfer ratio and myelin in postmortem multiple sclerosis brain. Ann Neurol 2004;56:407-15

19. van Buchem MA, Steens SC, Vrooman HA, et al. Global estimation of myelination in the developing brain on the basis of magnetization transfer imaging: a preliminary study. AJNR Am J Neuroradiol 2001;22:762-66

20. Xydis V, Astrakas L, Zikou A, et al. Magnetization transfer ratio in the brain of preterm subjects: age-related changes during the first 2 years of life. Eur Radiol 2006;16:215-20

21. Ge Y, Grossman RI, Babb JS, et al. Age-related total gray matter and white matter changes in normal adult brain. Part II. Quantitative magnetization transfer ratio histogram analysis. AJNR Am J Neuroradiol 2002;23:1334-41

22. Spilt A, Geeraedts T, de Craen AJ, et al. Age-related changes in normal-appearing brain tissue and white matter hyperintensities: more of the same or something else? AJNR Am J Neuroradiol 2005;26:725-29

23. Ropele S, Seewann A, Gouw AA, et al. Quantitation of brain tissue changes associated with white matter hyperintensities by diffusion-weighted and magnetization transfer imaging: the LADIS (Leukoaraiosis and Disability in the Elderly) study. J Magn Reson Imaging 2009;29:268-74

24. Hofman PA, Kemerink GJ, Jolles J, et al. Quantitative analysis of magnetization transfer images of the brain: effect of closed head injury, age and sex on white matter. Magn Reson Med 1999;42:803-06

25. Schmidt R, Enzinger C, Ropele S, et al. Progression of cerebral white matter lesions: 6-year results of the Austrian Stroke Prevention Study. Lancet 2003;361:2046-48

26. Fazekas F, Kleinert R, Offenbacher H, et al. Pathologic correlates of incidental MRI white matter signal hyperintensities. Neurology 1993:43:1683-89

27. Tofts PS, Davies GR, Dehmeshki JM. Histograms: measuring subtle diffuse disease. In: Tofts P, ed. Quantitative MRI of the Brain. Chichester, UK: John Wiley \& Sons Ltd: 2003:581-610

28. Ropele S, Fazekas F. Magnetization transfer MR imaging in multiple sclerosis. Neuroimaging Clin N Am 2009;19:27-36

29. Benedetti B, Charil A, Rovaris $M$, et al. Influence of aging on brain gray and white matter changes assessed by conventional, MT, and DT MRI. Neurology 2006;66:535-39

30. Cowell PE, Turetsky BI, Gur RC, et al. Sex differences in aging of the human frontal and temporal lobes. J Neurosci 1994;14:4748-55

31. Coffey CE, Lucke JF, Saxton JA, et al. Sex differences in brain aging: a quantitative magnetic resonance imaging study. Arch Neurol 1998;55:169-79

32. Xu J, Kobayashi S, Yamaguchi S, et al. Gender effects on age-related changes in brain structure. AJNR Am J Neuroradiol 2000;21:112-18

33. Sack I, Beierbach B, Wuerfel J, et al. The impact of aging and gender on brain viscoelasticity. Neuroimage 2009;46:652-57

34. de Leeuw FE, de Groot JC, Oudkerk M, et al. Hypertension and cerebral white matter lesions in a prospective cohort study. Brain 2002;125:765-72

35. Murray AD, Staff RT, Shenkin SD, et al. Brain white matter hyperintensities: relative importance of vascular risk factors in nondemented elderly people. Radiology 2005;237:251-57

36. Firbank MJ, Wiseman RM, Burton EJ, et al. Brain atrophy and white matter hyperintensity change in older adults and relationship to blood pressure: brain atrophy, WMH change and blood pressure. J Neurol 2007;254:713-21. Epub 2007 Apr 20

37. Gouw AA, van der Flier WM, Fazekas F, et al. Progression of white matter hyperintensities and incidence of new lacunes over a 3-year period: the Leukoaraiosis and Disability study. Stroke 2008;39:1414-20. Epub 2008 Mar 6

38. Nagai M, Hoshide S, Ishikawa J, et al. Ambulatory blood pressure as an independent determinant of brain atrophy and cognitive function in elderly hypertension. J Hypertens 2008;26:1636-41

39. Graham SJ, Henkelman RM. Understanding pulsed magnetization transfer. J Magn Reson Imaging 1997;7:903-12 